\title{
The Influence of Viscosity and Surface Curvature on the Pressure Distribution of a Stream Thrust Probe
}

\author{
Renee Pasman* \\ California Polytechnic State University, San Luis Obispo, CA, 93407 \\ $\&$ \\ Russell M. Cummings ${ }^{\dagger}$ \\ United States Air Force Academy, USAF Academy, CO, 80840
}

\begin{abstract}
Determining the local stream thrust (a vector quantity) from a measured pitot pressure (a scalar quantity) requires either knowledge of the flow direction, or a probe shape that compensates for flow direction. This compensation would ideally make the measured pressure directly proportional to the component of momentum along the probe axis. The flow angle sensitivity required to resolve this component of momentum was determined theoretically previously. A proposed probe nose shape was analyzed using CFD and found to produce flow angle sensitivity close to the required sensitivity. The proposed nose shape was also tested in a wind tunnel at Mach 1.67, 2.45, and 3.48 at angles of attack from 0 to 15 degrees. The test results indicate that the flow angle sensitivity of the proposed nose shape agrees with the required sensitivity to within $1 \%$ up to a flow angle of $15^{\circ}$. The current work extends the original theoretical development for the optimum nose shape to include viscous affects and surface curvature. The new second-order theory agrees well with experimental results for both the stream thrust probe as well as other, independent data. Further work can be done to refine the theory.
\end{abstract}

\section{Introduction}

T $\mathrm{N}$ many experimental evaluations of jet engines, the actual thrust of the engine can only be measured by taking reaction forces against a test stand. This technique measures the thrust minus the drag of the engine, but does not directly measure the thrust (as shown in Figure 1).

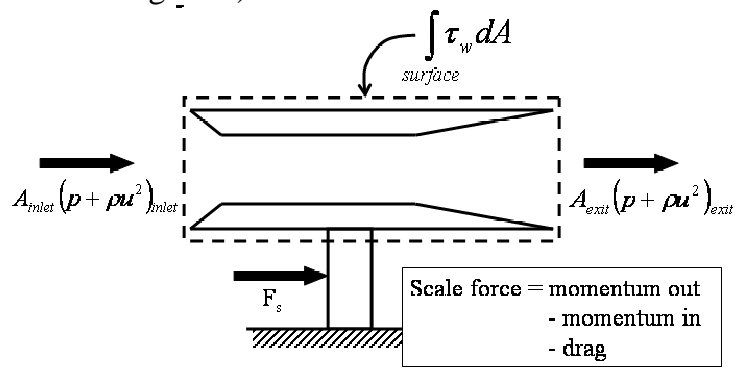

Probe-based stream thrust determination can provide a measure of gross or net thrust during frecjet testing. Scale force can determine only "thrust minus drag".

Figure 1. Static thrust measurements (Refs. 1 and 2).

A possible improvement to this method is to directly measure the thrust in the jet exhaust itself, as shown in Figure 2. This leads to the problem of how to measure the pressures in the exhaust even though portions of the exhaust

\footnotetext{
* Graduate Research Assistant, Aerospace Engineering Department, AIAA Student Member.

${ }^{\dagger}$ Professor, Department of Aeronautics, AIAA Associate Fellow.
} 
could be curving away from the nominal thrust direction by as much as 15 degrees. In several previous papers, ${ }^{1-3}$ a derivation was given to determine the local stream thrust from a measured pitot pressure. Ideal, perfect gas behavior was assumed to illustrate the technique, which requires a probe shape that automatically compensates for flow direction. ${ }^{2}$ A CFD study compared the flow angle compensation characteristics of two proposed probe geometries. ${ }^{2}$ The probe nose shape is designed such that the measured pressure is proportional to the component of momentum along the probe axis over a broad range of flow angles. This is in contrast to the typical requirement for a pitot probe to be insensitive to flow angle. If the probe were instead sensitive to the magnitude of the momentum vector, it would be required to also determine the flow angle in order to resolve the component in the desired direction. If the probe shape can automatically resolve the desired component of momentum, there would be no need to separately determine the flow angle.

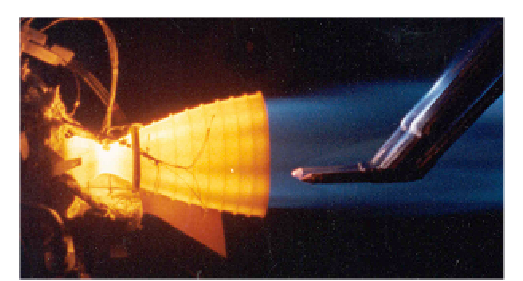

Figure 2. Pitot probe in the exhaust of a rocket engine.

\section{Desired Flow Angle Sensitivity}

Consider an axisymmetric pitot probe immersed in a flow of angle $\alpha$ as illustrated in Figure 3 . The pressuresensing orifice is on the axis of the probe at the probe tip.

Figure 3. Schematic of axi-symmetric probe.

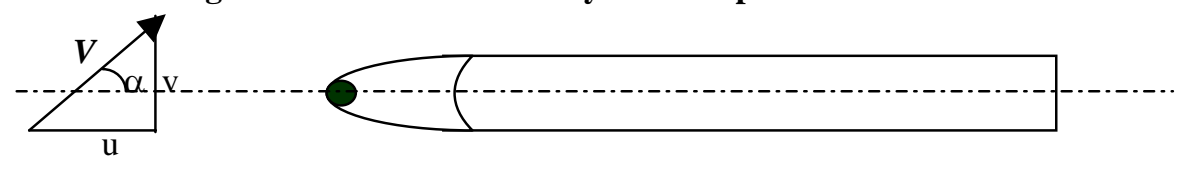

We desire the ratio of the stream thrust $\left(P+\rho u^{2}\right)$ to the pressure sensed by the orifice at the probe tip $\left(P_{m}\right)$ to be independent of the flow angle. That is, we require

$$
\frac{P+\rho u^{2}}{P_{m}} \neq f(\alpha)
$$

Note that $u$ is the component of velocity along the axis of the probe, whereas $v$ is the component normal to the probe axis. The probe is intended to sense the component of momentum along the probe axis. If the vector $\boldsymbol{V}$ denotes the flow velocity, then $u$ is given by

$$
u=|\boldsymbol{V}| \cos \alpha
$$

so

$$
u^{2}=|\boldsymbol{V}|^{2} \cos ^{2} \alpha
$$

Thus, the requirement for flow angle independence can be written by combining Eqns. 1 and 3 as ${ }^{1}$

$$
\frac{P+\rho|\boldsymbol{V}|^{2} \cos ^{2} \alpha}{P_{m}} \neq f(\alpha)
$$


where the simplified notation $P_{\alpha}$ is used to indicate the sensed orifice pressure as a function of the flow angle $\alpha$ and $P_{0}$ is the pitot pressure sensed when the probe is aligned with the flow (note that $P_{m}$ is also a function of flow angle). It was shown in Ref. 1 that the required flow angle sensitivity is given by

$$
\frac{P_{\alpha}}{P_{0}}=\left[\frac{1+\gamma M^{2} \cos ^{2} \alpha}{1+\gamma M^{2}}\right]
$$

The desired distribution given by Eqn. 5 is shown in Fig. 4.

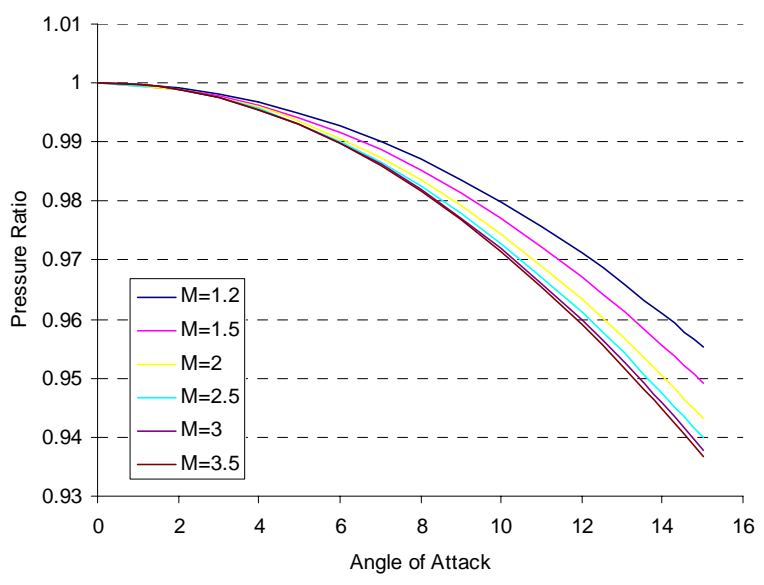

Figure 4. Desired supersonic distribution.

The problem is now to determine the probe nose geometry that best approximates this dependence. To calculate engine thrust within the required margins, the optimized probe nose geometry must give results within approximately $0.1 \%$ of the desired distribution shown in Fig. 4.

\section{Second-Order Boundary Layer Theory}

When testing the probe model discussed in Hiers et al. ${ }^{1,2,3}$ in CFD simulations, a consistent difference of around one percent was noticed between inviscid-flow predictions and the experimental data. To explain this difference, two main factors were examined. The preliminary CFD predictions and the theory developed by Hiers et al. ${ }^{1,2}$ assumed the flow was inviscid, which precluded the existence of a boundary layer. However, real flow (such as the experimental data) has a boundary layer and viscous effects. Therefore, the effect of a boundary layer had to be examined theoretically. At first, it was believed that a boundary layer could not lead to a significant pressure difference, due to the fact that the pressure gradient across the boundary layer is assumed to be zero in laminar flow over a thin flat plate. However, due to the large Reynolds' numbers experienced by the probe, it is more likely that the boundary layer is turbulent, rather than laminar. Based on White's Viscous Fluid Flow, ${ }^{4}$ a turbulent boundary layer, whether incompressible or compressible, produces a slight pressure gradient normal to the boundary layer. However, again according to White, this difference usually accounts for only around a $0.4 \%$ difference in the pressure. A second factor contributing to a change in pressure had to be considered.

The probe surface is highly curved, as shown in Fig. 3. Based on the work by Sislian, ${ }^{5}$ streamline curvature produces unexpectedly large changes in boundary layer properties. In a turbulent boundary layer surface curvature primarily affects the turbulent mixing energy. Also, the convex curvature of the probe surface causes additional centrifugal acceleration. According to Gillis et al., ${ }^{6}$ the problem of measuring static pressure is more complicated because it is a function of the coordinate normal to the curved surface. Wilcox shows these effects occur even for boundary layers where $\delta$ is much smaller than the radius of curvature, $R$, and at high Reynolds numbers. ${ }^{7}$ White also gives a relationship for the pressure gradient normal to the surface as a function of local curvature: ${ }^{4}$

$$
\frac{\partial P}{\partial y}=\frac{\rho u^{2}}{R}
$$


Other researchers have taken a different approach. Seginer ${ }^{11}$ found that a compressible boundary layer would, in most cases, have a normal pressure gradient too large to ignore, and developed a quasi-similar solution for these cases. Melnik et al. ${ }^{12}$ developed the theory behind viscous transonic flows over airfoils at high Reynolds numbers, with special attention being paid to the curvature effects.

To include the effects of curvature and a resulting normal pressure gradient, it is necessary to expand on the standard first-order boundary layer theory. Prandtl's first-order boundary-layer theory does not account for surface curvature, and it also precludes the existence of a normal pressure gradient. Higher-order boundary layer theories, such as the second-order theory given in Schlichting ${ }^{13}$ do account for these effects. By using a second-order theory, the effects of curvature and displacement would be accounted for. These effects are often negligible, but in this case, they could cause the small difference between the experimental and CFD results. For more detail on previous work in curved boundary layers, the reader is referred to the review performed by Pasman. ${ }^{16}$

Finally, a justification for approximating a supersonic flow field with a subsonic theory is required. Supersonic flow over a blunt object will, at supersonic freestream speeds, produce a detached shock wave in front of the body. Error! Reference source not found., reproduced here as Figure, shows a schematic of the situation. Given this transonic flow scenario, several statements can be made. Forward of the point, normal to freestream point, there exists a normal shock. Away from this point, "the shock wave gradually becomes curved and weaker, eventually evolving into a Mach wave at large distances from the body."17 For part of the flow, the shock is strong enough to induce subsonic flow behind it, which leads to subsonic surface flow on part of the probe.

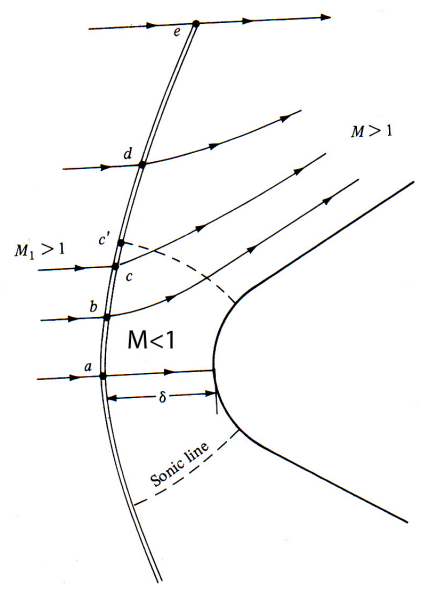

Figure5. Supersonic flow over a blunt object (Ref. 17).

\section{A. Second-order Boundary Layer Theory}

Given the inability of first-order boundary layer theory to predict the effects of the boundary layer on a highly curved surface, a second-order theory was explored. For this section, much of the information came from $\mathrm{H}$. Schlichting's Boundary Layer Theory, especially Chapters VII and IX. ${ }^{13}$ For more detail, the reader is referred to Schlichting ${ }^{13}$ and Pasman. ${ }^{16}$

To obtain a theory accurate to the second order, it is required to use a singular perturbation scheme, to obtain asymptotic expansions to the Navier-Stokes solutions for high Reynolds numbers. For this theory, the perturbation scheme is:

$$
\varepsilon=\frac{1}{\sqrt{\operatorname{Re}}}=\frac{1}{\sqrt{\frac{U_{\infty} R_{o}}{v}}}
$$

The term in the denominator is the Reynolds number with the nose radius of curvature at the stagnation point as the characteristic length. Using this perturbation scheme allows the boundary layer theory to be extended to higher orders. The first terms in this solution are exactly Prandtl's first-order solution. Further terms can be interpreted "as corrections to the classical theory which represent boundary layer effects of second order," ${ }^{, 13}$ such as curvature.

The starting point of the second-order theory is the curvilinear Navier-Stokes equations, stated as: ${ }^{13}$ 


$$
\begin{aligned}
& \frac{\partial u}{\partial t}+\frac{R}{R+y} u \frac{\partial u}{\partial x}+v \frac{\partial u}{\partial y}+\frac{v u}{R+y}=-\frac{R}{R+y} \frac{1}{\rho} \frac{\partial p}{\partial x}+v\left\{\frac{R^{2}}{(R+y)^{2}} \frac{\partial^{2} u}{\partial x^{2}}+\frac{\partial^{2} u}{\partial y^{2}}+\frac{1}{R+y} \frac{\partial u}{d y}-\frac{u}{(R+y)^{2}}+\right. \\
& \left.\frac{2 R}{(R+y)^{2}} \frac{\partial v}{\partial x}-\frac{R}{(R+y)^{3}} \frac{d R}{d x} v+\frac{R y}{(R+y)^{3}} \frac{d R}{d x} \frac{\partial u}{\partial x}\right\} \\
& \frac{\partial v}{\partial t}+\frac{R}{R+y} u \frac{\partial v}{\partial x}+v \frac{\partial v}{\partial y}-\frac{u^{2}}{R+y}=-\frac{1}{\rho} \frac{\partial p}{\partial y}+v\left\{\frac{\partial^{2} v}{\partial y^{2}}-\frac{2 R}{(R+y)^{2}} \frac{\partial u}{\partial x}+\frac{1}{R+y} \frac{\partial v}{\partial y}+\frac{R^{2}}{(R+y)^{2}} \frac{\partial^{2} v}{\partial x^{2}}-\right. \\
& \left.\frac{v}{(R+y)^{2}}+\frac{R}{(R+y)^{3}} \frac{d R}{d x} u+\frac{R y}{(R+y)^{3}} \frac{d R}{d x} \frac{\partial v}{\partial x}\right\} \\
& \frac{R}{R+y} \frac{\partial u}{\partial x}+\frac{\partial v}{\partial y}+\frac{v}{R+y}=0
\end{aligned}
$$

The curvilinear coordinate system is shown in Figure 5.

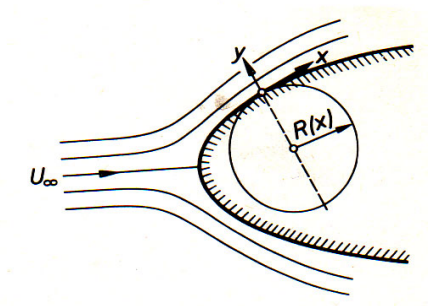

Figure 5. Curvilinear coordinate system (from Ref. 13).

These equations assume two-dimensional, incompressible flow, with high Reynolds numbers. Both the high Reynolds number assumption and the two-dimensional flow assumption hold for the flow over the stream thrust probe. The results will have to be corrected for compressibility at the end of the calculations, assuming that the flow behind the nearly normal shock of the probe is subsonic in the region of interest. For the derivation, all lengths are made non-dimensional by reference to the radius of curvature at the stagnation point, $R_{o}$. Velocities are referenced to the free steam velocity, and pressures to $\rho U_{\infty}^{2}$. For details of the derivation, the reader is referred to Refs. 13 and 16.

Schlichting describes a simple second-order theory solution to Eqn. 8 as:

$$
c_{p}=\frac{1}{1+1.38 x^{*}}
$$

where $x^{*}=x^{\prime} / R_{o}$ and $x^{\prime}$ is the Cartesian $x$-axis. The theoretical results from Eqn. 9 are plotted versus the desired and experimental distributions in Figure 6 for a stream thrust probe that was tested at the U.S. Air Force Academy's Tri-Sonic Wind Tunnel.

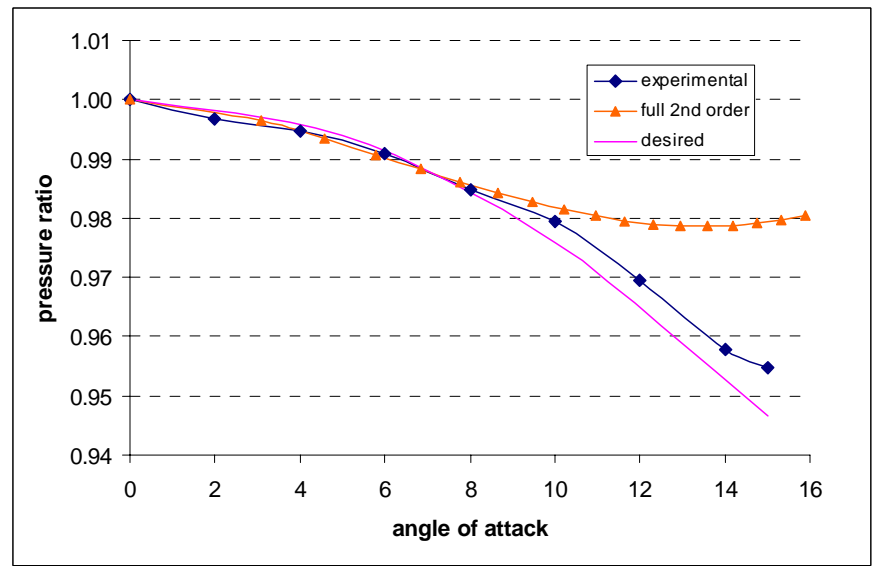

Figure 6. Schlichting second-order theory comparison.

American Institute of Aeronautics and Astronautics 
As can be seen in the above figure, the theory agrees very well with the experimental and desired results up to an angle of attack of 7 degrees, after which it deviates greatly. This is caused by the fact that $K$ (which is defined as $K=R_{o} / R(x)$ and is a measure of the surface curvature away from the stagnation point) is not included in the derivation of the theory. A new second-order theory therefore had to be derived, to include $K$. The derivation is summarized here, and for further details of this derivation the reader is referred to Ref. 16.

To re-derive the pressure coefficient equation, the same procedure was used as in Schlichting (Ref. 13) following the methods of Ref. 14. The following assumptions were made for $U, u$ and $v$ :

$$
\begin{aligned}
& U(x, 0)=U_{11} x+\varepsilon U_{21} x+O\left(\varepsilon^{2}\right) \\
& u(x, y, \varepsilon)=U_{11} x f^{\prime}(\eta)+\varepsilon\left[\sqrt{U_{11}} x F_{c}^{\prime}(\eta)+U_{21} x F_{d}^{\prime}(\eta)\right]+O\left(\varepsilon^{2}\right) \\
& v(x, y, e)=-\varepsilon \sqrt{U_{11}} f(\eta)-\varepsilon^{2}\left[F_{c}(\eta)-\eta f(\eta)+U_{21} F_{d}(\eta) / \sqrt{U_{11}}\right]+O\left(\varepsilon^{3}\right)
\end{aligned}
$$

Where the new variable is defined as: $\eta=\sqrt{U_{11}} N=\sqrt{U_{11}} y / \varepsilon$, since $N=y / \varepsilon$.

When these assumptions are substituted into the second-order boundary layer equations, and $K$ is left as a variable, the following equations result:

$$
\begin{aligned}
& f^{\prime \prime \prime}+f f^{\prime \prime}+1-f^{\prime 2}=0 \\
& F_{c}^{\prime \prime \prime}+f F_{c}^{\prime \prime}-2 f^{\prime} F_{c}^{\prime}+f^{\prime \prime} F_{c}=K \eta f^{\prime \prime \prime}+K f^{\prime \prime}+K \eta f f^{\prime \prime}+K f f^{\prime}-\eta f f^{\prime \prime} \\
& F_{d}^{\prime \prime \prime}+f F_{d}^{\prime \prime}-2 f^{\prime} F_{d}^{\prime}+f^{\prime \prime} F_{d}=-2
\end{aligned}
$$

The first-order equation is unchanged. The second-order solutions have again been split into two partial solutions. The $F_{c}$ equation can be further simplified using the first-order equation:

$$
F_{c}^{\prime \prime \prime}+f F_{c}^{\prime \prime}-2 f^{\prime} F_{c}^{\prime}+f^{\prime \prime} F_{c}=K \eta\left(1-f^{\prime 2}-\frac{f^{\prime \prime}}{\eta}-\frac{f f^{\prime}}{\eta}+\frac{f f^{\prime \prime}}{K}\right)
$$

These equations were solved using a fourth-order Runge-Kutta solver, using the Bisection method to determine the missing boundary conditions. Using the results from the solver allowed the pressure coefficient equation to be expressed in a form that included $K$ :

$$
c_{p}=1-U_{11}^{2} x^{2}\left(1+\varepsilon\left(\frac{2 K^{*} A}{\sqrt{U_{11}}}-\frac{2 U_{21}}{U_{11}}\right)\right)
$$

where $A$ is a constant determined by a fourth-order Runge-Kutta solver (for the current problem $A=1.8805$ ). $U_{11}$ and $U_{2 I}$ are two constants whose numerical value depends on the shape of the nose of the probe. The $x$ value in this equation is the curvilinear $x$, which is tangent to the surface of the probe. This value can be determined from the Cartesian $x$ and $y$ coordinates using Pythagorean's Theorem. The $x$ values are then non-dimensionalized with the nose radius of curvature, $R_{o}$.

This equation is derived from the incompressible Navier-Stokes equation. To correct for compressibility, the Prandtl-Glauert correction was used. The values for the coefficient $U_{11}$ and $U_{2 I}$ are also different for the current probe, compared to those for the more slender probe analyzed by Schlichting. With $U_{11}=3.0$ and $U_{2 I}=-0.61$, the pressure distribution shown in Figure 7 results for a freestream Mach number of 1.67. 


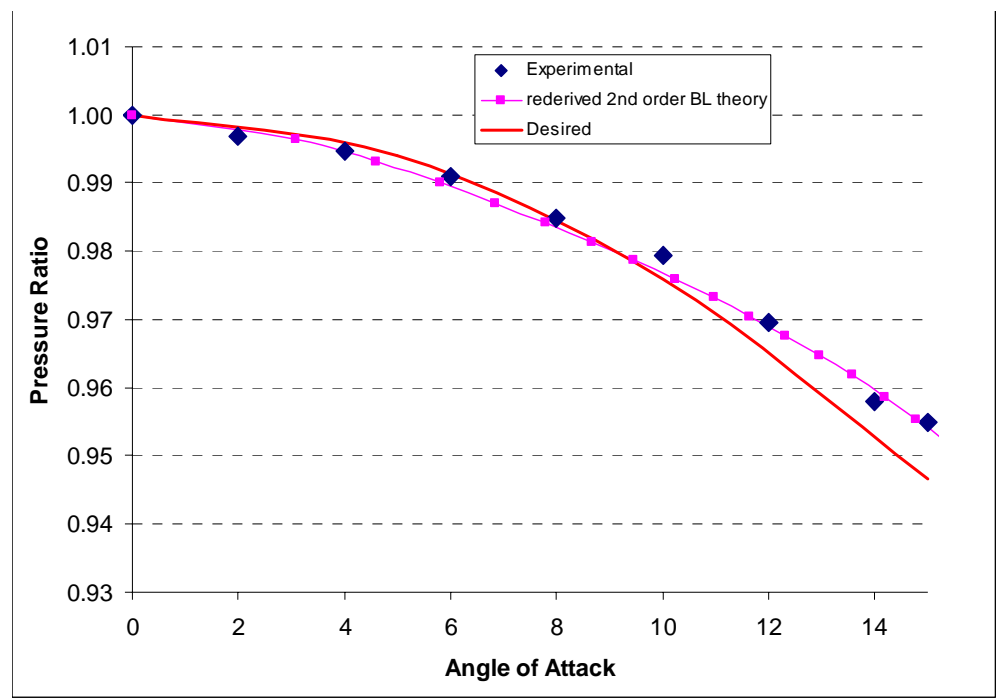

Figure 7. New second-order pressure distribution.

As seen in Figure 7, the newly derived second-order theory agrees well with the experimental results.

Two notes on the theory must be made. First, the value of $U_{11}$ must be determined for a specific shape. Neither Schlichting ${ }^{13}$ nor Devan ${ }^{15}$ provide any guidance as to how to select the appropriate value of $U_{11}$ for an arbitrary shape. A comparison of several different shapes and their published $\mathrm{U}_{11}$ values can begin to establish guidelines to selecting an appropriate constant. The two shapes for which Schlichting ${ }^{13}$ gives $\mathrm{U}_{11}$ values are the parabola solved in Schlichting, ${ }^{13}$ and the Rankine Oval analyzed in Devan. ${ }^{15}$ One of the differences between the three nose shapes is the radius of curvature at the nose, $R_{o}$. The current stream thrust probe design is the bluntest, whereas Schlichting's parabola is the sharpest. Based on the nose radius of curvature and given $U_{11}$ values, a relationship can be determined to predict $\mathrm{U}_{11}$ values for a given shape, as shown in Figure 8.

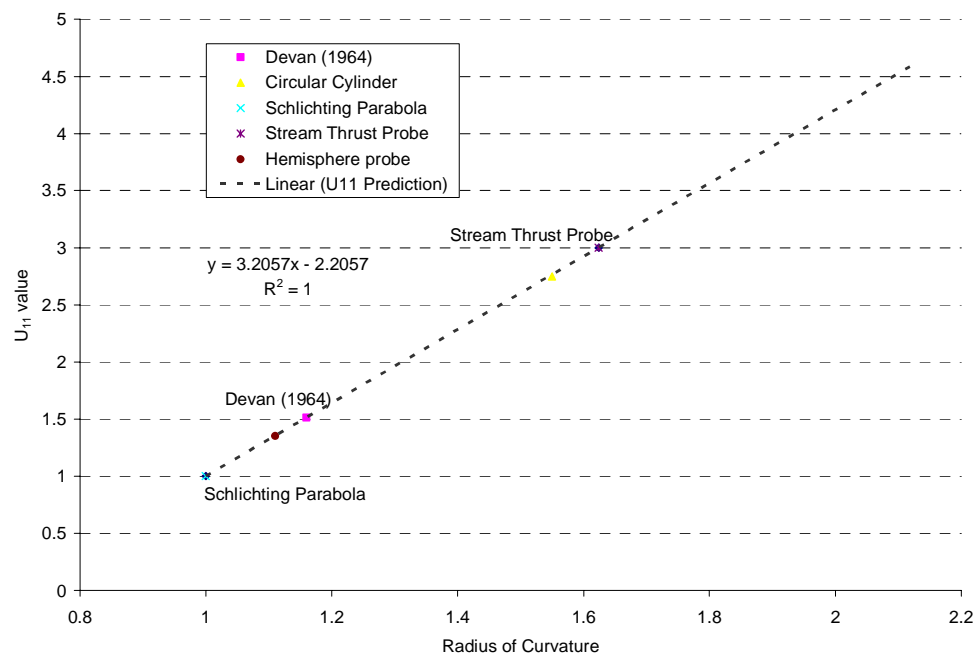

Figure 8. Relationship for $U_{11}$.

For a first approximation, a simple linear relation between the two known points was established, and additional points were extrapolated from it.

The second note which must be made relates to the incompressible nature of the theory. To justify using an incompressible theory in a flow field that is clearly compressible, it is necessary to consider the flow field directly in front of the probe nose. A bow shock forms in front of the probe as the freestream flow goes supersonic. The bow shock is detached from the surface of the probe, due to the blunt nose. The flow at the probe nose, and the pressure sensing orifice encounters, is therefore subsonic. This infers that the flow behavior may be due more to subsonic effects than supersonic effects. Following this line of thinking, the curvature of the probe tip takes on special 
significance, since subsonic flow is known to be very sensitive to surface curvature. Then as the shock curves, it becomes weaker, and the flow the probe encounters becomes supersonic. However, because the area of interest falls only at plus/minus 15 degrees angle of attack, it is assumed the flow behind the shock in this area is subsonic.

\section{Comparison to Experimental Results}

To test the results of the current second-order theory, comparisons were made to other existing experimental data sets. The first was a hemispherical probe nose, and the second an infinite circular cylinder. For more details on these cases, the reader is referred to Pasman. ${ }^{16}$

\section{A. Hemispherical Probe Nose}

In 1954, Stalder and Nielsen published experimental heat transfer results for a hemispherical probe with spikes of different lengths. ${ }^{18}$ Their results, along with the second-order theory results are shown in Fig. 9.

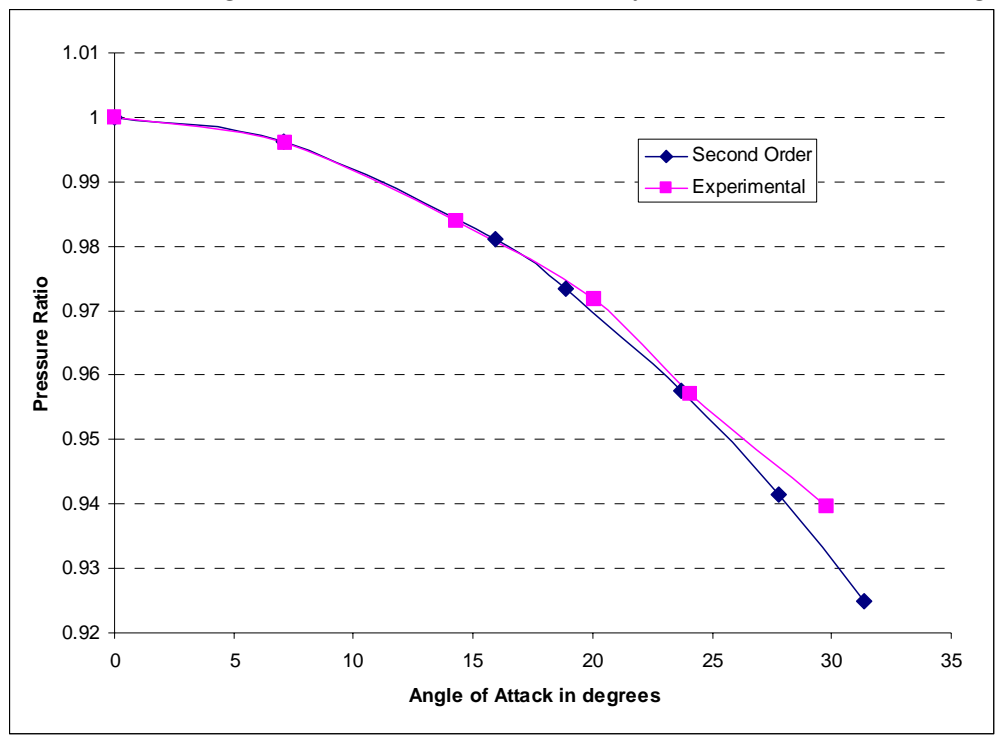

Figure 9. Comparison of Stalder and Nielsen results.

As seen, for the desired angle of attack range of zero to 20 degrees, the two distributions agree very well. Even for angles of attack higher than 20 , the distributions are very similar.

\section{B. Circular Cylinder}

In Schlichting's $8^{\text {th }}$ edition, Figure 1.18 gives the surface pressure distribution for an infinite circular cylinder in near-hypersonic flow ( $\mathrm{M}=4.02)$. These results, along with the second-order theory results are shown in Figure 10.

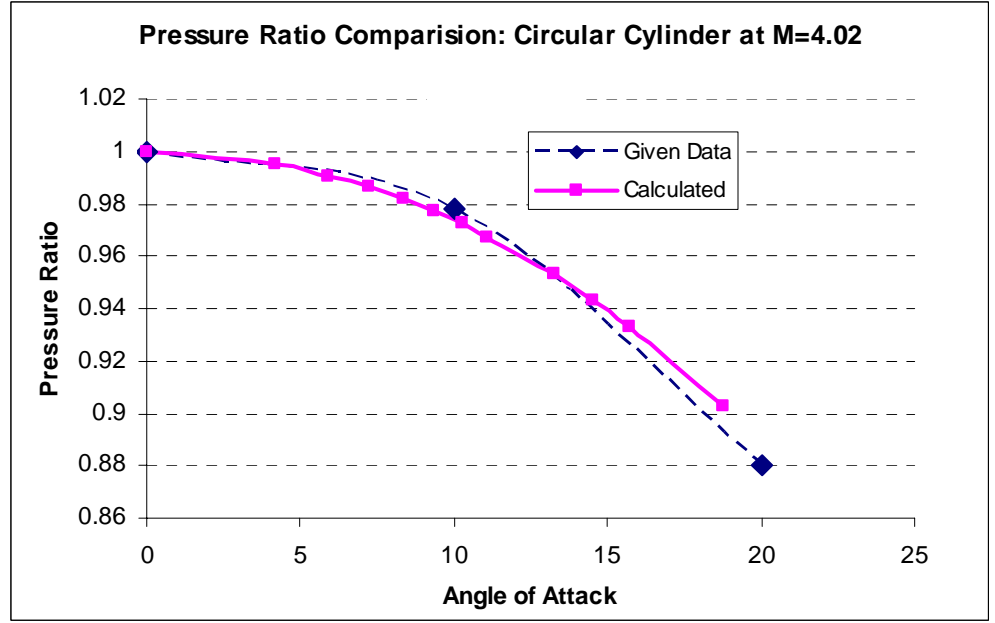

Figure 10. Comparison of Schlichting results.

American Institute of Aeronautics and Astronautics 
For both these experimental data sets, the second-order theory compares well with the experimental results. In Spring 2005, a second round of experimental testing was conducted at the Air Force Academy, on a new stream thrust probe, with a nose geometry based on inviscid CFD predictions. The results were found to not be in close agreement with the desired distribution. As seen in Figure 11 below, second-order theory predicted this outcome for this shape.

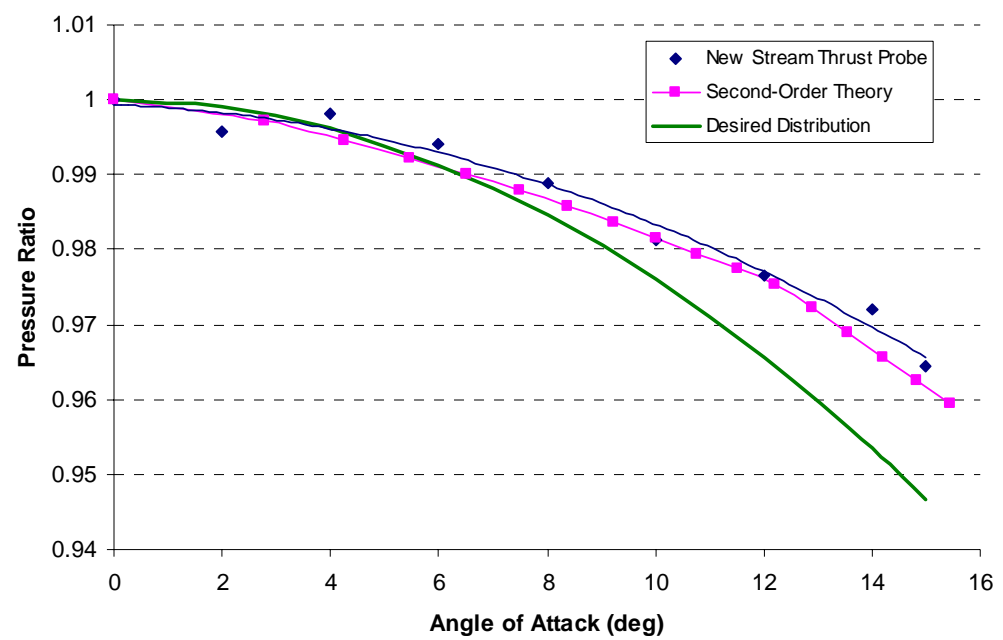

Figure 11. Comparison with USAFA experiments.

Overall, second-order theory agrees well with the experimental datasets. With the overall accuracy of the theory confirmed, the theory can be used to predict the optimum shape for a stream thrust probe.

\section{Application to Stream Thrust Probe}

The second-order theory can also be used to predict the optimum shape for the stream thrust probe. Assume that the stream thrust probe nose geometry is described by the super-ellipse equation: ${ }^{19}$

$$
y= \pm r\left[1+\left|\frac{x-a}{a}\right|^{m}\right]^{1 / n}
$$

The variables $m$ and $n$ control the shape of the nose. By varying these two geometric factors, a large number of candidate shapes can be evaluated fairly quickly. To determine the optimum probe shape, first the effects of $m$ and $n$ on the bluntness of the probe were studied. Next, the effects of changing the bluntness of the probe on the pressure distribution over the probe were studied. By automating a large part of this process, many different probe shapes could be studied and compared. Based on these trends, one optimized probe was found at $m=3.55, n=2.2$. The resulting shape and pressure distribution is shown in Figure 12, where the comparison to the desired trend from Eqn. 5 is excellent. 

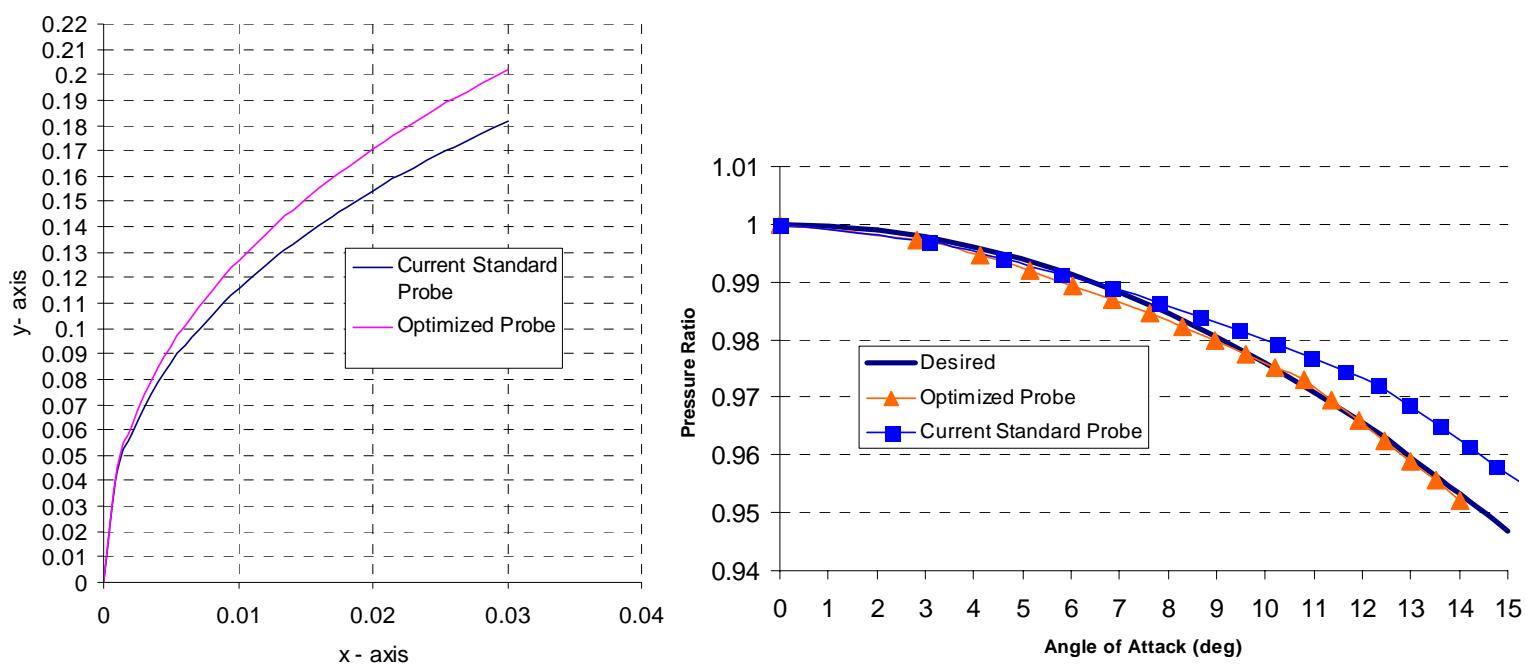

Figure 12. Optimized probe shape and pressure distribution.

Besides this probe, several other combinations of $m$ and $n$ were found to give close agreement to the desired distribution. These results are summarized in Table 1 below.

Table 1. Additional optimized probes compared to desired distribution.

\begin{tabular}{|l|l|l|l|}
\hline$m$ & 3.55 & 3.43 & 3.5 \\
\hline$n$ & 2.165 & 2.23 & 2.2 \\
\hline Max error & $0.1366 \%$ & $0.1639 \%$ & $0.1532 \%$ \\
\hline Avg error & $-0.0468 \%$ & $-0.0110 \%$ & $-0.0397 \%$ \\
\hline
\end{tabular}

The error given in the above table shows the maximum and average deviation from the desired distribution. All of these probes have a maximum error of approximately $0.15 \%$ of the desired distribution, and a average error of less than $0.05 \%$, meaning they would allow for accurate thrust measurements. Further tests are required to determine which of these probes is the "most optimum". Factors such as manufacturability must also be considered. Wind tunnel tests would probably be required to determine the best probe.

\section{Conclusions}

The purpose of this paper was to present a second-order boundary layer theory to determine the pressure distribution over a highly curved stream thrust probe. After the accuracy of the theory was confirmed by comparing its results to known experimental data sets, the theory was used to predict a family of stream thrust probes which closely match the previously determined desired distribution.

Recommendations for future work fall into two categories: further work on the theory, and further work on the stream thrust project. The theory currently used is adapted from an incompressible, laminar second-order theory. A compressibility correction is used to account for compressible flow, and turbulence is not addressed. A better solution would be to develop a compressible second-order theory and to find some way to account for turbulence. Also, further work could be put into determining the constants required for the various shapes. While the current linear method is adequate, it is simplistic. A more precise method would improve the accuracy of the overall theory.

Based on the second-order theory presented here, several new probe shapes have been put forward for consideration. These probe shapes should be tested, both in CFD and experimentally. If the greater accuracy predicted continues to be seen in these tests, one or several of these shapes should be considered for the final stream thrust probe. Attention needs to be paid to the feasibility of constructing these probes to the desired precision needed to produce the correct shape. The difficulty of producing the probe accurately may become a limiting factor in which probe shape is finally chosen.

Overall, the second-order theory appears to correctly predict the surface pressure distribution on the stream thrust probe. Accounting for viscosity and the curvature of the probe tip, and the resulting changes in the flow field give the correct theoretical results, with a maximum error of approximately $0.1 \%$ compared to the experimental results. 


\section{Acknowledgments}

The authors would like to thank the support of Robert Hiers and James Sirbaugh of the Arnold Engineering Developing Center, as well as Major Barrett McCann of the U.S. Air Force Academy, and Dr. Colleen Kirk and Dr. David Marshall, both from California Polytechnic State Univesity. Finally, we would like to thank Prof. H.T. Yang of the Aerospace Engineering Department at the University of Southern California for reviewing our theoretical development.

\section{References}

${ }^{1}$ Hiers, R.S., and Pruitt, D.W., "Determination of Thrust from Pitot Pressure Measurements," AIAA Paper 2001-3314, July 2001.

${ }^{2}$ Hiers, R.S., and Sirbaugh, J.R., "The Aerodynamics of Stream Thrust Probes," AIAA Paper 2003-1092, Jan. 2003.

${ }^{3}$ Hiers, R.S., Sirbaugh, J.R., McCann, B.T., Cummings, R.M., Luft, L.S., Grillos, D.P., "Experimental Verification of the Aerodynamics of Stream Thrust Probes," AIAA Paper 2005-1215, Jan. 2005.

${ }^{4}$ White, F.M., Viscous Fluid Flow, New York: McGraw-Hill, 1974.

${ }^{5}$ Sislian, J.P., "Equations of Motion and Two-Equation Turbulence Model for Plane or Axisymmetric Turbulent Flows in Body-Oriented Orthogonal Curvilinear Coordinates and Mass-Averagaed Dependent Variables," NASA CR 3025, Aug. 1978.

${ }^{6}$ Gillis, J.C., Johnston, J.P., Moffat, R.J., Kays, W.M., "Experimental Data and Model for the Turbulent Boundary Layer on a Convex, Curved Surface," NASA CR 3391, Mar. 1981.

${ }^{7}$ Wilcox, D.C., Chambers, T.L., "Streamline Curvature Effects on Turbulent Boundary Layers," AIAA Journal, Vol. 15. No. 4, 1977, pp. 574-580.

${ }^{8}$ Gallimore, S.J., "Spanwise Mixing in Multistage Axial Flow Compressors: Part II - Throughflow Calculations Including Mixing," Journal of Transactions of the ASME, Vol. 108, July 1986, pp. 10-16.

${ }^{9}$ Novak, R.A., Hearsey, R.M, "A Nearly Three-Dimensional Intrablade Computing System for Turbomachinery," Journal of Transactions of the ASME, Vol. 99, March 1977, pp. 154-163.

${ }^{10}$ Barger, R.L., Brooks, C.W. Jr., "A Streamline Curvature Method for Design of Supercritical and Subcritical Airfoils," NASA TN-D-7770, Sep. 1974.

${ }^{11}$ Seginer, A., "Compressible Boundary Layer with Normal Pressure Gradients: Quasi-Similarity Equations Their Properties at the Wall and at Sharp and Blunt Leading Edges," AIAA Paper 72-696, June 1972.

${ }^{12}$ Melnik, R.E., Chow, R., Mead, H.R., "Theory of Viscous Transonic Flow over Airfoils at High Reynolds Number," AIAA Paper 77-680, Jan. 1977.

${ }^{13}$ Schlichting, H. Boundary Layer Theory, $7^{\text {th }}$ Ed. New York: McGraw-Hill, 1979.

${ }^{14}$ Van Dyke, M. Perturbation Methods in Fluid Mechanics. Stanford: Parabolic Press, 1975.

${ }^{15}$ Devan, L. Second-order Incompressible Laminar Boundary Layer Development on a Two-Dimensional SemiInfinite Body. Ph. D. Thesis, University of California, Los Angeles, 1964.

${ }^{16}$ Pasman, R., "Theoretical Calculation of Stream Thrust Probe Surface Pressure Distributions Including the Effects of Viscosity and Curvature," M.S. Thesis, California Polytechnic State University, San Luis Obispo, June 2005.

${ }^{17}$ Anderson Jr., J.D., Fundamentals of Aerodynamics, $3{ }^{\text {rd }}$ Edition, New York: McGraw-Hill, 2001

${ }^{18}$ Stalder, J.R. and Nielsen, H.V., "Heat Transfer from a Hemisphere-cylinder Equipped with Flow-separation Spikes," NACA TN 3287, 1954.

${ }^{19}$ Hiers, R. S. "Super-Ellipses as a Parametric Shape for Stream Thrust Probes," AEDC Memorandum (unpublished), May 2004. 